\title{
Conducting polymers forward
}

\author{
Twenty years after the Nobel Prize in Chemistry for the discovery of conducting polymers, we reflect on the open \\ research questions and the status of commercial development of these materials.
}

O ften, in science, breakthroughs happen by making the most of mistakes. A good example of this is Hideki Shirakawa, Alan MacDiarmid and Alan Heeger's discovery that organic polymers are able to transport electric current $^{1}$, which led to them sharing the 2000 Nobel Prize in Chemistry ${ }^{2}$.

According to Shirakawa's recount, in their studies on acetylene polymerization he and his collaborator Hyung Chick Pyun accidentally used a concentration of catalysts that was a thousand times too high, obtaining a silver film composed of crystalline fibres. Shirakawa continued to experiment on the chemistry of polyacetylene films, trying to transform them into graphite by exposure to halogen vapours - he paid less attention, though, to what was happening to their electrical properties. MacDiarmid, who met Shirakawa at a seminar in Tokyo, invited him to the University of Pennsylvania to perform those experiments with Heeger. Together, they observed a conductivity increase by various orders of magnitude (pictured), which marked the official entrance of conjugated polymers into the realm of conducting materials. The journey from that lucky accident to current research and commercialization efforts of semiconducting and highly conducting polymers is described by Xugang Guo and Antonio Facchetti in a Comment in this issue.

In the years following the initial discovery, the investigation of the mechanisms leading to this increased conductivity continued. The halogenation process that the trio performed was an example of p-doping: halogens oxidize the polyacetylene chains by extracting electrons, which leaves positive charges free to move under an applied electric field. Small molecules, too, proved to be effective in oxidizing or reducing the polymer chains and making them transport positive or negative charges, respectively. Alternative doping processes, such as the use of acids to protonate the polymers backbone, have been developed, and recent studies have also shown that charges can be directly transferred between neutral polymers ${ }^{3}$.

A better understanding of the connections between molecular and electronic structure allowed chemists to synthesize undoped polymers with semiconducting properties. The demonstration that these organic

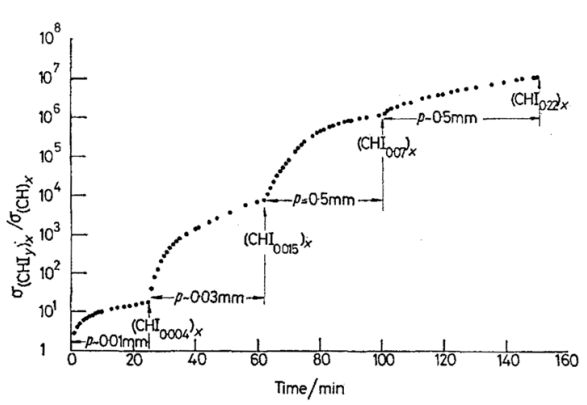

Impact of iodine vapours on the conductivity of polyacetylene. Reproduced from ref. ${ }^{1}$, RSC.

semiconductors could be used in transistors and even emit light through charge injection ${ }^{4}$ further boosted research in the field, prompting the synthesis of polymers with tailored optoelectronic properties. A few of these materials are now produced at scale and used in various applications, yet the path to commercial success is narrow. As Guo and Facchetti highlight, the polymerization, isolation and purification processes adopted, the cost of monomers and reagents, and the management of toxic by-products are all factors determining the economic competitiveness of these materials. Equally important is compatibility with industrial manufacturing processes, which further imposes restrictions on the solvents used, their rheological properties and shelf stability.

Plenty is still unknown about the properties of conjugated polymers, in particular regarding charge transport and how it is affected by the polymer morphology. Charge motion is very fast along the backbone of a polymer chain, yet it slows down when charges hop between neighbouring chains, as Henning Sirringhaus and colleagues described in a recent Review ${ }^{5}$. Torsion and folding of the chains, changes in crystallinity and other forms of static and dynamic disorder impact the way charges travel and the mobility achieved in organic semiconductors.

Yet some degree of disorder in polymer films can be beneficial, as it helps penetration of dopant molecules and other ionic species. Polymers are indeed not only electronic conductors; they can also transport ions that, importantly, can modulate the electronic behaviour of the material. For example, in a Letter published in this issue, Scott Keene and collaborators showed that the electronic output of a neuromorphic device made from an ion-responsive conjugated polymer can be controlled by the dopamine released by cells cultured on the polymer, realizing a biohybrid synaptic connection. Ionicelectronic interactions, however, further complicate the understanding of these materials: as recently examined by Jonathan Rivnay and colleagues ${ }^{6}$, ionic-electronic injection, transport and coupling play an important role in the behaviour of organic mixed ionic-electronic conductors.

In the race for materials commercialization, researchers have explored application spaces where the combination of good electrical and mechanical properties, as well as the versatile processability of conducting polymers, could be a winner. Antistatic coatings, organic light-emitting panels, flexible photovoltaic modules and organic thin-film transistors are some of the applications that have gone far along the commercialization path. The mixed ionic-electronic conduction properties of some conducting polymers are used in electrochromic devices, and their biocompatibility may prove advantageous in other areas such as implantable bioelectronics, biochemical sensing and health monitoring.

The discovery of electrical conductivity has endowed conjugated polymers with incredible appeal. Once mainly a playground for chemists, materials scientists and process engineers, they are now one of the preferred materials of investigation for physicists, electronic engineers, bioengineers and more. We look forward to seeing whether such collaborations will lead to new breakthroughs, and possibly new Nobel prizes.

Published online: 20 August 2020 https://doi.org/10.1038/s41563-020-0792-7

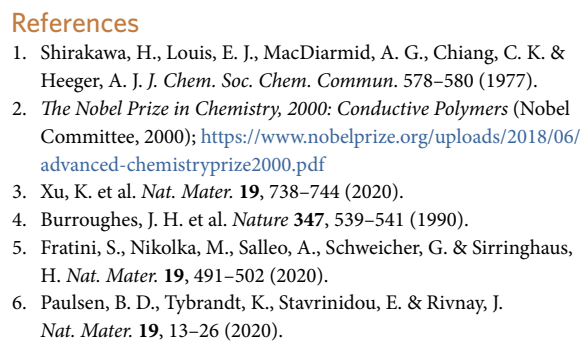

